\title{
Paraffin Nanocomposites for Heat Management of Lithium-Ion Batteries: A Computational Investigation
}

\author{
A. H. N. Shirazi, ${ }^{1,2}$ Farzad Mohebbi, ${ }^{2}$ M. R. Azadi Kakavand, ${ }^{2}$ B. He, ${ }^{2}$ and T. Rabczuk ${ }^{1,2}$ \\ ${ }^{1}$ Institute of Research \& Development, Duy Tan University, Quang Trung, Da Nang, Vietnam \\ ${ }^{2}$ Institute of Structural Mechanics, Bauhaus-Universität Weimar, Weimar, Germany
}

Correspondence should be addressed to A. H. N. Shirazi; ali.hn.s@outlook.com and T. Rabczuk; timon.rabczuk@uni-weimar.de

Received 25 September 2015; Accepted 2 February 2016

Academic Editor: Zafar Iqbal

Copyright (c) 2016 A. H. N. Shirazi et al. This is an open access article distributed under the Creative Commons Attribution License, which permits unrestricted use, distribution, and reproduction in any medium, provided the original work is properly cited.

\begin{abstract}
Lithium-ion (Li-ion) batteries are currently considered as vital components for advances in mobile technologies such as those in communications and transport. Nonetheless, Li-ion batteries suffer from temperature rises which sometimes lead to operational damages or may even cause fire. An appropriate solution to control the temperature changes during the operation of Li-ion batteries is to embed batteries inside a paraffin matrix to absorb and dissipate heat. In the present work, we aimed to investigate the possibility of making paraffin nanocomposites for better heat management of a Li-ion battery pack. To fulfill this aim, heat generation during a battery charging/discharging cycles was simulated using Newman's well established electrochemical pseudo-2D model. We couple this model to a 3D heat transfer model to predict the temperature evolution during the battery operation. In the later model, we considered different paraffin nanocomposites structures made by the addition of graphene, carbon nanotubes, and fullerene by assuming the same thermal conductivity for all fillers. This way, our results mainly correlate with the geometry of the fillers. Our results assess the degree of enhancement in heat dissipation of Li-ion batteries through the use of paraffin nanocomposites. Our results may be used as a guide for experimental set-ups to improve the heat management of Li-ion batteries.
\end{abstract}

\section{Introduction}

In recent years, communication technologies have been rapidly progressed. Therefore, increasing the efficiency is inevitable in applications ranging from portable electronics to renewable energies and power plant is inevitable. In portable electronics, the battery plays a crucial role in their efficiency. On the other hand, there are some deficiencies in the application of the present batteries in which the thermal management of battery packs is one of the most important problems. In some cases, uncontrollable temperature inside the batteries may result in fire or even explosion. Recently, Goli and coworkers [1] proposed the utilization of paraffin phase change material (PCM) with graphene fillers to enhance the performance of lithium-ion (Li-ion) batteries versus intense self-heating. The above-mentioned methodology describes a heat storage-heat conduction approach that has affirmative effects on thermal management of any types of batteries including Li-ion batteries. Kumaresan et al. [2] assessed discharge efficiency prediction of Lithiumion cell at various operating temperatures $\left(15-45^{\circ} \mathrm{C}\right)$ by a thermal model. Numerical results were compared with experimental data obtained from lithium-ion pouch cells. In addition, Goyal and Balandin [3] investigated the thermal properties of some materials with the hybrid graphenemetal particle fillers. In this research activity, the thermal conductivity of composites was measured with the variation of temperature. The achieved results are applicable for the thermal management of electronics and optoelectronics. Zolot et al. [4] presented the hybrid vehicle test focusing on battery thermal management. The results of tests proved that the performance of battery packs significantly changes due to thermal condition. Yeow et al. [5] developed 3D finite element (FE) models with consideration of geometry variations in order to model the treatment of Lithium ion cells of vehicle electrification applications. The results show that the 3D electrothermal model satisfactorily describes the electrothermal behaviour of the Li-ion battery cells and 
the results are in acceptable agreement with battery temperature measurements. To simulate various cell types for an electric drive system, Benger et al. [6] developed a parameterized model for the electrochemical and thermal properties. The comparison between modelling results and the measurement indicates that the developed model can predict the current voltage behaviour and the temperature development of the various cells. Mohammadian et al. [7] made a comparison between internal and external cooling procedures for thermal management of LIBs. They performed 2D and 3D transient thermal analysis of a prismatic Li-ion battery cell. The results showed that, with the same pumping power, external cooling decreases the bulk temperature less than internal cooling and, moreover, internal cooling considerably decreases the standard deviation of the temperature inside the battery.

It is quite well-known that the phase change materials can store a large amount of heat according to their high capacity of latent heat storage. They can absorb high amount of heat while their temperature remains nearly constant. According to their applications, there are various types of PCMs that each of them is applicable for certain temperature ranges [8]. However, the properties of PCMs yet can be varied by changing their chemical composition or by adding additional components. In this regard, one solution is to fabricate nanocomposites structures through adding nanoscale fillers to PCMs. For the application in Li-ion batteries, the thermal conductivity is the main parameter to improve. Carbon based nanostructures such as graphene and carbon nanotubes offer the highest thermal conductivities available in the nature [9]. Therefore, they could be considered as the best candidates to enhance heat conduction for PCMs. Shahil and Balandin [10] showed that adding multilayer graphene to commercial grease can enhance drastically the thermal conductivity of the mixture even in low fillers volume percent. There exist numerous theoretical and experimental studies available in the direction of composite materials with superior thermal and mechanical properties [11-17].

In this paper, we investigate the temperature rise in a Lithium ion battery pack. In this regard, the simulations are used to provide a general viewpoint to guide experiments which are expensive and time consuming as well $[18,19]$. We used pure and nanocomposites paraffin PCMs to dissipate the heat produced during the charge/discharge cycles of a battery pack containing 16 individual Li-ion batteries. Fullerene, graphene, and CNT were used as fillers with different volumes concentration to enhance the thermal conductivity of paraffin hybrid phase change material. The effects of various volume percent of fillers on thermal behaviour of batteries were investigated at different charging/discharging rates (C-rates). Our results show remarkable effect of using paraffin PCMs on the heat dissipation of a battery pack. Thermal conductivity coefficients of hybrid paraffin PCMs were obtained through finite element modelling of representative volume elements. Heat generation rates during the charging/discharging cycles were simulated using Newman's well established electrochemical pseudo-2D model [20]. It is shown that as the filler's volume fraction inside the paraffin increases, the amount of heat dissipation to the ambient environment increases, which is due to the increase in the thermal conductivity of PCMs.

\section{Theory and Modeling}

Numerical simulations of electrochemical response of Li-ion battery at different charging/discharging cycles were simulated within the framework of the pseudo-2D electrochemical model proposed by Newman and Thomas-Alyea [20]. In this model, the dynamic performance of a cell is characterized by the solution of four partial differential equations describing the time evolution of the lithium concentration profile in the electrode and electrolyte phases, under charge conservation. Then, based on the electrochemical response, heat generation was calculated which was used in the heat transfer model for the evaluation of temperature rises in a battery pack. Accordingly, we first present the electrochemical model which is considered as a $1 \mathrm{D}$ problem. Then, we discuss the heat sources and temperature evolution in the battery cell. We note that the heat sources are calculated based on the $1 \mathrm{D}$ electrochemical problem, which is then coupled with $3 \mathrm{D}$ heat transfer modelling. Figure 1 illustrates the $1 \mathrm{D}$ Li-ion cell model which consists of three main regions: the mesocarbon microbead (MCMB) negative composite electrode (graphite type structure), an electron-blocking separator, and $\mathrm{LiCoO}_{2}$ positive composite electrode.

Lithium concentration in the electrolyte phase is obtained by using Fick's second law along the $x$-coordinate with a source term coupled to the local reaction current density which yields the following equation:

$$
\frac{\partial\left(\varepsilon_{e} C_{e}\right)}{\partial t}=\frac{\partial}{\partial x}\left(D_{e}^{\mathrm{eff}} \frac{\partial C_{e}}{\partial x}\right)+\frac{1-t_{+}^{0}}{F} j^{\mathrm{Li}},
$$

where $\varepsilon_{e}$ is the volume fraction of electrolyte, $C_{e}$ is the concentration of Li in electrolyte, $D_{\text {eff }}$ is the effective diffusion coefficient of $\mathrm{Li}$ in the electrolyte, $t_{+}^{0}$ is the transference number of Li-ions with respect to the velocity of solvent, and $F$ is the Faraday constant. $j^{\mathrm{Li}}$ is the reaction current density.

The Li-ions cannot diffuse through the current collectors, as set by the boundary conditions in (2), which is valid at the two electrode/current collector interfaces

$$
\left.\frac{\partial C_{e}}{\partial x}\right|_{x=0}=\left.\frac{\partial C_{e}}{\partial x}\right|_{x=L}=0 .
$$

At the interfaces between the positive electrode/separator and separator/negative electrode, the concentration of the binary electrolyte $\left(C_{e}\right)$ and its flux $\left(\partial C_{e} / \partial x\right)$ are continuous.

For the modelling of diffusion of Li-ions inside the solid particles, Newman's model assumes the electrode can be described by a lattice of spherical particles of identical size representing the intercalation centre into which metallic lithium diffuses. The distribution of lithium in the solid phase $\left(C_{s}\right)$ is described by Fick's second law of diffusion in polar coordinates $(r)$, as shown in the following relation:

$$
\frac{\partial C_{s}}{\partial t}=\frac{D_{s}}{r^{2}} \frac{\partial}{\partial r}\left(r^{2} \frac{\partial C_{s}}{\partial r}\right)
$$

where $D_{s}$ is the diffusion coefficient in solid particles. The solution is constrained by a zero gradient boundary condition at the centre of the particle from symmetry arguments, 


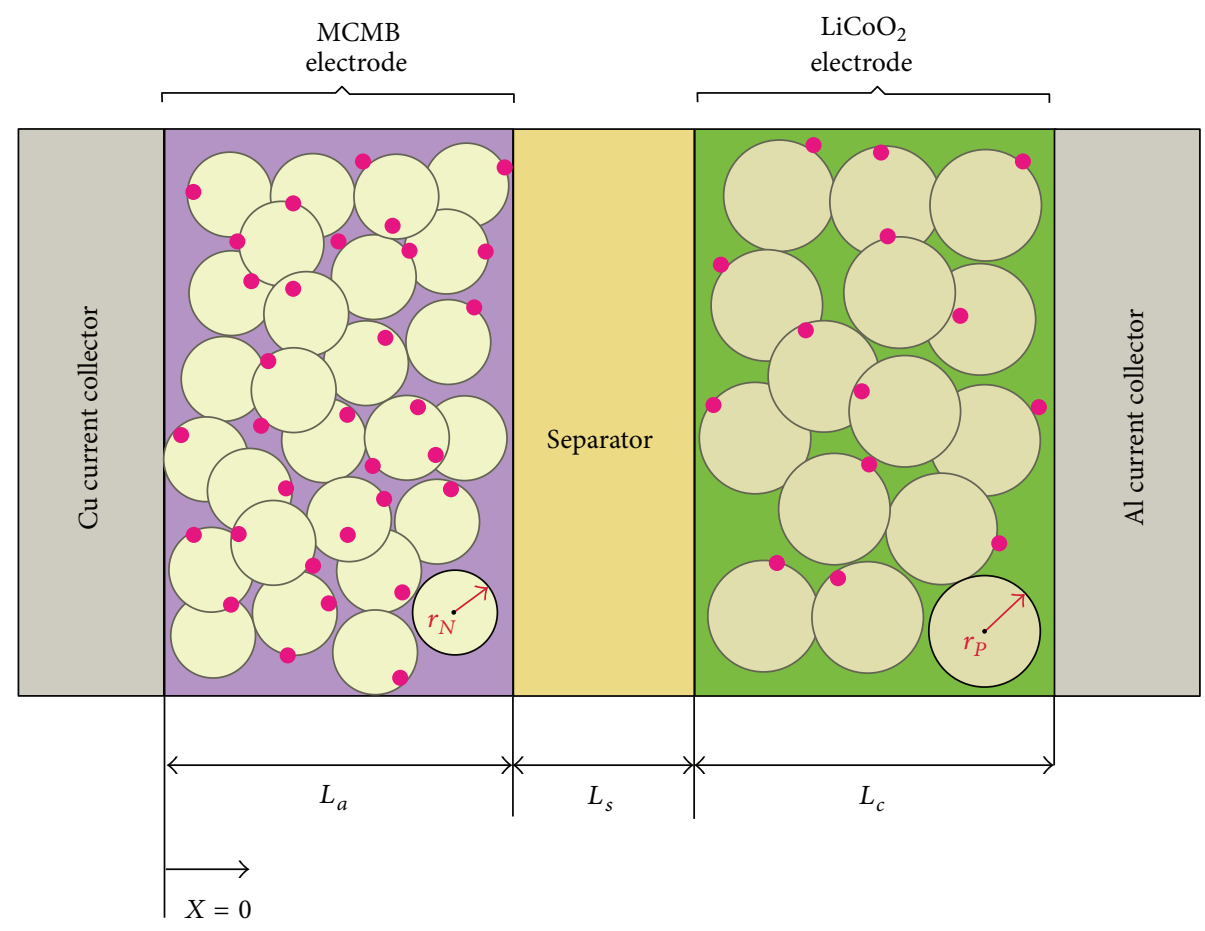

FIGURE 1: Schematic illustration of Newman's electrochemical model based on 1D ( $x$-direction) electrochemical cell model coupled with 1D microscopic ( $r$-direction) solid diffusion model.

while at the surface of the particle the lithium flux must correspond to the reaction current density $j^{\mathrm{Li}}$, as imposed by the following equation:

$$
\begin{gathered}
\left.\frac{\partial C_{s}}{\partial r}\right|_{r=0}=0, \\
-\left.D_{s} \frac{\partial C_{s}}{\partial r}\right|_{r=R_{s}}=\frac{j^{\mathrm{Li}}}{a_{s} F},
\end{gathered}
$$

where $r$ is the particle radius of negative or positive electrodes. Potential in the electrolyte phase $\phi_{e}$ is a function of reaction current density $j^{\mathrm{Li}}$ and the local concentration of $\mathrm{Li}$ $\left(C_{e}\right)$ by the following relation:

$$
\frac{\partial}{\partial x}\left(k^{\mathrm{eff}} \frac{\partial \phi_{e}}{\partial x}\right)+\frac{\partial}{\partial x}\left(k_{D}^{\mathrm{eff}} \frac{\partial}{\partial x} \ln C_{e}\right)+j^{\mathrm{Li}}=0,
$$

where $k^{\text {eff }}$ is the effective ionic conductivity of electrolyte and $k_{D}^{\text {eff }}$ is the effective diffusional conductivity of a specie. The solution of (5) is subject to a zero gradient boundary condition at the two current collector/electrode interfaces

$$
\left.\frac{\partial \phi_{e}}{\partial x}\right|_{x=0}=\left.\frac{\partial \phi_{e}}{\partial x}\right|_{x=L}=0 .
$$

Finally, the potential in the solid phase $\phi_{s}$ is introduced as a function of the conductivity of the electrode $\sigma^{\text {eff }}$ and the reaction current density $j^{\mathrm{Li}}$, as described by the following relation:

$$
\frac{\partial}{\partial x}\left(\sigma^{\mathrm{eff}} \frac{\partial \phi_{s}}{\partial x}\right)=j^{\mathrm{Li}}
$$

The boundary conditions are then introduced as follows: at the electrode/separator interface, there is no flux of charge, and thus a zero gradient boundary condition is applied. Moreover, at the electrode/current collector interface, the charge flux corresponds to the current in the outside circuit

$$
\begin{gathered}
-\left.\sigma_{-}^{\mathrm{eff}} \frac{\partial \phi_{s}}{\partial x}\right|_{x=0}=\left.\sigma_{+}^{\mathrm{eff}} \frac{\partial \phi_{s}}{\partial x}\right|_{x=L}=\frac{I}{A}, \\
\left.\frac{\partial \phi_{s}}{\partial x}\right|_{x=\delta-}=\left.\frac{\partial \phi_{s}}{\partial x}\right|_{x=L-\delta+}=0 .
\end{gathered}
$$

Last, but not the least, $j^{\mathrm{Li}}$ which is a parameter that simulate charge transfer rate in all previous four PDEs is determined by the Bulter-Volmer equation as follows:

$$
j^{\mathrm{Li}}=a_{s} i_{0}\left\{\exp \left[\frac{0.5 F}{R T} \eta\right]-\exp \left[-\frac{0.5 F}{R T} \eta\right]\right\},
$$

where $a_{s}$ is the active surface area per electrode unit volume $\left(\mathrm{m}^{2} / \mathrm{m}^{-3}\right), F$ is the Faraday constant, $T$ is temperature, $R$ is the universal gas constant, and finally $i_{0}$ is exchange current density which is introduced as follows:

$$
i_{0}=K_{i}\left(C_{e}\right)^{0.5}\left(C_{s, \max }-C_{s, \text { surf }}\right)^{0.5}\left(C_{s, \text { surf }}\right)^{0.5},
$$

where $K_{i}$ is the reaction rate coefficient, $C_{s, \max }$ is the maximum $\mathrm{Li}$ concentration in the solid phase particles, $C_{s, \text { surf }}$ is the concentration of $\mathrm{Li}$ at the surface of solid phase particles, and $C_{e}$ is the Li-ion concentration in electrolyte. The overpotential $\eta$ in (9) is given by

$$
\eta=\phi_{s}-\phi_{e}-U,
$$


TABLE 1: Parameters for 1D lithium ion battery cell [2].

\begin{tabular}{|c|c|c|c|c|}
\hline Parameter & Description & Negative electrode & Separator & Positive electrode \\
\hline$\overline{t^{0}}$ & Transference number of electrolytes & 0.435 & 0.435 & 0.435 \\
\hline$L$ & Length of electrode $(\mu \mathrm{m})$ & 73.5 & 25 & 70 \\
\hline$r$ & Solid particle radius $(\mu \mathrm{m})$ & 12.5 & - & 8.5 \\
\hline$\varepsilon_{s}$ & Solid phase volume fraction & 0.5052 & - & 0.55 \\
\hline$\varepsilon_{e}$ & Electrolyte phase volume fraction & 0.4382 & 0.45 & 0.30 \\
\hline$\varepsilon_{b}$ & Binder volume fraction & 0.0566 & - & 0.15 \\
\hline$D_{s}$ & Solid phase diffusion coefficient $\left(\mathrm{m}^{2} / \mathrm{s}\right)$ & $1.4523 \times 10^{-13}$ & - & $1.0 \times 10^{-11}$ \\
\hline$\rho$ & Active material density $\left(\mathrm{kg} / \mathrm{m}^{3}\right)$ & 2292 & - & 5031.67 \\
\hline$C_{s, \max }$ & Maximum concentration in solid phase $\left(\mathrm{mol} / \mathrm{m}^{3}\right)$ & 31858 & - & 49943 \\
\hline SOC & State of charge in charge/discharge (\%) & $5 / 95$ & - & $95 / 50$ \\
\hline$C_{l, 0}$ & Initial electrolyte concentration $\left(\mathrm{mol} / \mathrm{m}^{3}\right)$ & 1000 & 1000 & 1000 \\
\hline brug & Bruggeman coefficient for tortuosity & 4.1 & 2.3 & 1.5 \\
\hline$k_{i}$ & Reaction rate coefficient at $25^{\circ} \mathrm{C}$ & $1.764 \times 10^{-11}$ & - & $6.6667 \times 10^{-11}$ \\
\hline$K_{T}$ & Thermal conductivity $(\mathrm{W} /(\mathrm{m} \cdot \mathrm{K}))$ & 1.7 & 0.16 & 2.1 \\
\hline$\alpha_{a}, \alpha_{c}$ & Charge transfer coefficients & $0.5,0.5$ & & $0.5,0.5$ \\
\hline$\sigma$ & Solid phase conductivity (S/m) & 100 & - & 10 \\
\hline
\end{tabular}

where $U$ is the equilibrium potential which is the function of intercalated $\mathrm{Li}$ and is on the basis of empirical functions. The parameters used in the presented equations are listed in Table 1 which are on the basis of the work by Kumaresan et al. [2]. In addition, the equilibrium potentials for solid electrodes and electrolyte ionic conductivity as a function of Li concentration were all adopted from [2]. To calculate the effective ionic conductivity $\left(k_{\text {eff }}\right)$ and lithium diffusion coefficients $\left(D_{\text {eff }}\right)$ in the electrolyte in the different parts of the cell, we used the Bruggeman approximation as follows:

$$
\begin{aligned}
& k_{\text {eff }}=k_{i} \varepsilon^{\text {brug }_{i},} \\
& D_{\text {eff }}=D_{e} \varepsilon^{\text {brug }_{i},}
\end{aligned}
$$

where index $i$ refers to different cell regions (anode, separator, or cathode) and the exponent (brug) is the Bruggeman exponents (the values are listed in Table 1). The total generated heat is taken as the sum of reaction and joule (ohmic) heats. Typically, heat generation in lithium-ion batteries can be attributed to three main sources: heat from the reaction current and overpotentials $\left(q_{r}\right)$, ionic ohmic heat from the motion of lithium/lithium-ions through the solid $\left(q_{j}\right)$, and reversible heat $\left(q_{\mathrm{rev}}\right)$. In this study, these sources of heat are expressed as follows:

$$
\begin{aligned}
q_{r} & =A \int_{0}^{L} j^{\mathrm{Li}}\left(\phi_{s}-\phi_{e}-U\right) d x \\
q_{j} & =A \int_{0}^{L} \sigma^{\mathrm{eff}}\left(\frac{\partial \phi_{s}}{\partial x}\right)^{2} d x \\
q_{\mathrm{rev}} & =j^{\mathrm{Li}} T \frac{\partial U}{\partial T}
\end{aligned}
$$

Equation (15) presents the reversible heat generation which can be either positive or negative. This is related to the entropy changes in solid electrode materials which are referred to as $\partial U / \partial T$. As discussed in [2], the reversible heat plays

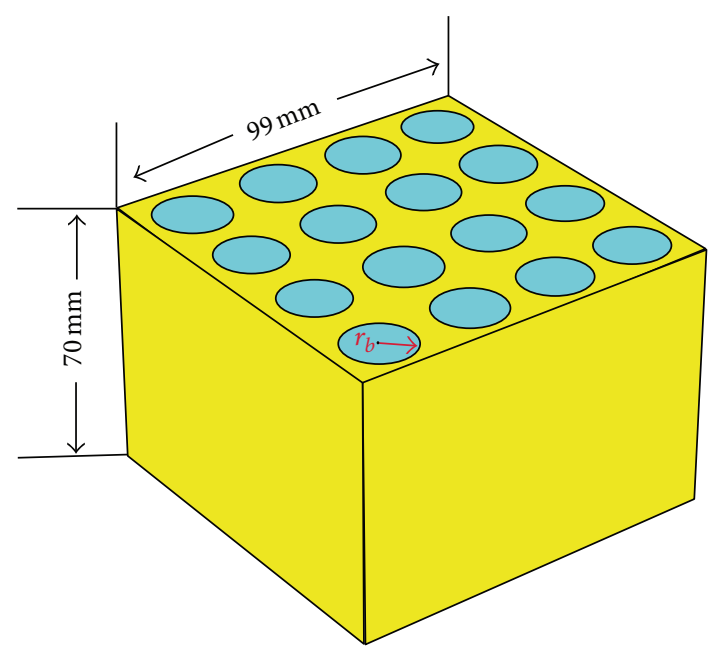

FIGURE 2: 3D heat transfer model coupled with electrochemical model to evaluate the temperature rises during the battery charging/discharging cycles. The Li-ion batteries and paraffin based structures are illustrated by blue and yellow colors, respectively. The battery radius, $r_{b}$, was chosen to be $9.2 \mathrm{~mm}$. The outer surface of the model is exposed to air.

an important role in the $\mathrm{LiCoO}_{2}$ electrode. In the present work, $\partial U / \partial T$ curves, as a function of $\mathrm{Li}$ concentration in solid particles, were adopted from [2]. We note that the coupled electrochemical heat transfer models have also been developed in numerous previous works [21-27].

The calculated heat generation rates based on Newman's model were then used to simulate the temperature rises in a battery pack containing 16 individual batteries. The heat transfer model is shown in Figure 2. This model includes two materials: Li-ion batteries and paraffin based structures. The material parameters used in the simulation are given in Table 2. The heat capacity of paraffin nanocomposites 


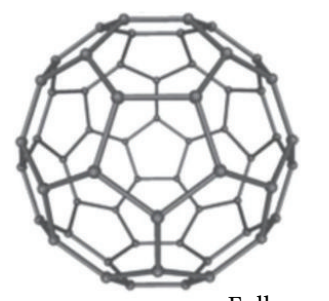

Fullerene

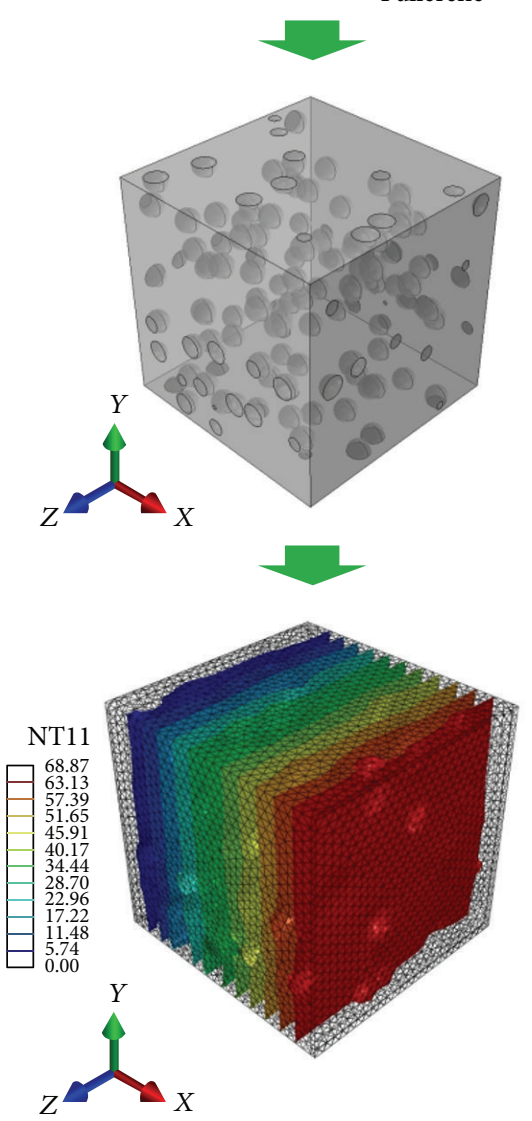

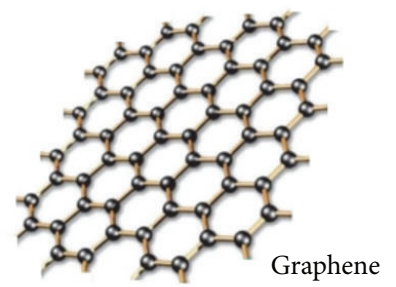

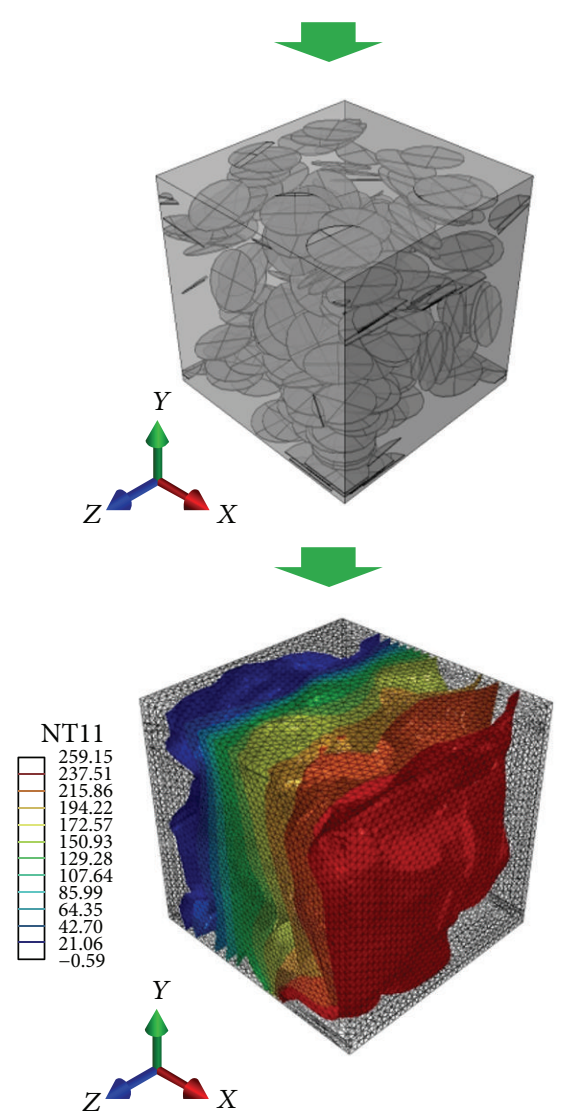

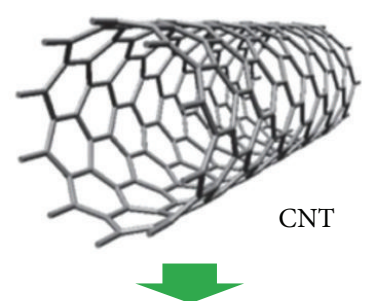

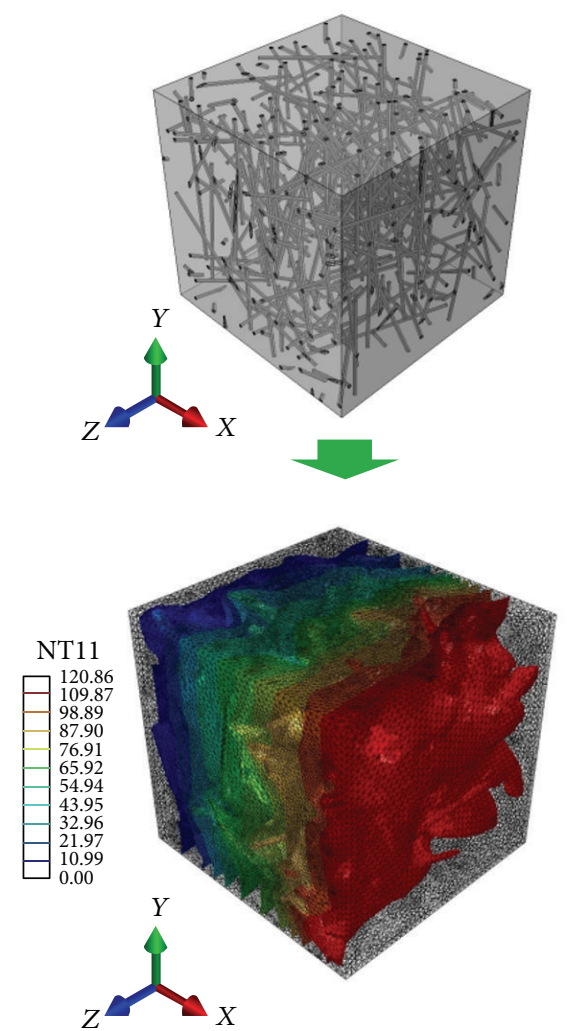

FIGURE 3: Different structures of nanofillers used in this study. We studied the thermal conductivity of paraffin nanocomposites filled with fullerene, graphene, and carbon nanotubes (CNT). Under each filler, the constructed finite element model in Abaqus is shown with 5\% volume concentrations for filler. Under each finite element model, the temperature distribution obtained from the application of the heat flux is plotted.

TABLE 2: Material properties.

\begin{tabular}{lccc}
\hline Material & Density $\left(\mathrm{kg} / \mathrm{m}^{3}\right)$ & $\begin{array}{c}\text { Heat capacity } \\
(\mathrm{J} /(\mathrm{kg} \cdot \mathrm{K}))\end{array}$ & $\begin{array}{c}\text { Thermal } \\
\text { conductivity } \\
(\mathrm{W} /(\mathrm{m} \cdot \mathrm{K}))\end{array}$ \\
\hline Battery cell & 2680 & 1280 & 1 \\
Paraffin & 900 & 2500 & 0.25 \\
Fullerene & 2200 & 717 & 3000 \\
CNT & 2200 & 717 & 3000 \\
Graphene & 2200 & 717 & 3000 \\
\hline
\end{tabular}

was also calculated using the rule of mixtures. The outer surfaces of $3 \mathrm{D}$ heat transfer model were exposed to air with convective heat transfer coefficient of $2.5 \mathrm{~W} / \mathrm{m}^{2} \cdot \mathrm{K}$.
The coupled electrochemical and heat transfer models were built in COMSOL/Multiphysics package using Li-ion battery and heat transfer modules.

In this work, we also studied the effective thermal conductivity of paraffin nanocomposites. Here, we include three different fillers of graphene, carbon nanotubes, and fullerene to study the thermal conductivities using the finite element approach. Computational limits of finite element method impose restrictions on the maximum number of elements used in a model. Thus, the simulations of composite materials are limited to the modeling of a representative volume element (RVE) of the system. In an attempt to construct the RVEs in a status closer to those in experimentally fabricated random composites, the 3D fillers were randomly distributed and oriented in the RVE. The atomic structures of three studied fillers along with the FE models constructed in 


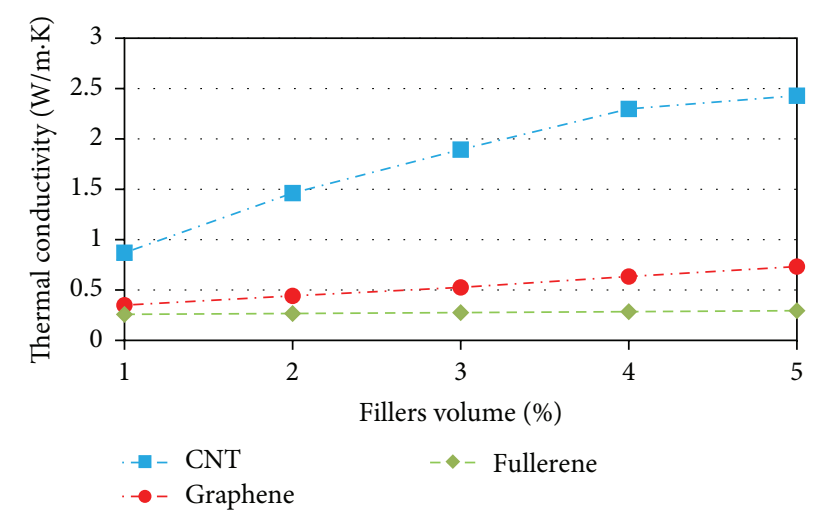

FIgURE 4: Calculated effective thermal conductivity of paraffin nanocomposites as a function of different fillers volume concentration.

ABAQUS software are illustrated in Figure 3. The RVEs were modeled in such a way that they satisfy the periodicity criterion. This means that if a filler is cut by a boundary face of RVE, the remaining part of that particle should continue from the opposite face. The random RVEs were constructed in ABAQUS by developing Python scripts as input files. We developed advanced $\mathrm{C}++$ codes for creating randomly distributed/oriented fillers with high volume concentrations and without intersection [28, 29]. In our FE modeling, we assumed perfect heat transfer condition (no interfacial resistance) between the fillers and the paraffin matrix. For the evaluation of thermal conductivity, we merged two thin auxiliary parts to the RVE as discussed in our previous work [29]. We then applied a constant heat flux along the sample and based on the established temperature profile the effective thermal conductivity was evaluated which is discussed in detail in [29]. We note that length to diameter ratio for CNT (with cylindrical geometry) and diameter to thickness ratio for graphene (defined with disc geometry) were both assumed to be 50 . The thermal conductivity of all fillers was assumed to be $3000 \mathrm{~W} / \mathrm{m} \cdot \mathrm{K}$ [30]. It is worth noting that this thermal conductivity may not be realistic for fullerene. Nevertheless, based on previous theoretical investigation [11], for spherical particles the thermal conductivity of composite does not correlate strongly with the fillers to matrix, contrast in properties.

\section{Results and Discussions}

Figure 4 depicts the finite element results for effective thermal conductivity of paraffin nanocomposites as a function of different filler's volume concentration. It is worthy to note that in our finite element modeling, for each RVE, we calculated the effective conductivities along three Cartesian directions. For each volume concentration, three or four RVEs were constructed with different filler distributions. To obtain converged effective thermal conductivity, the results for different RVEs as well as different directions were all averaged. Our results shown in Figure 4 reveal that the thermal conductivity of paraffin can be drastically improved by the addition of CNT. In addition, it is shown that the addition of fullerene leads to insignificant enhancement of effective thermal conductivity. In composite materials, the heat flux is transferred between matrix and filler through their contacting surfaces. Therefore, the filler's surface to volume ratio plays an important role in the final reinforcement. This can justify the least reinforcement in thermal conductivity by the addition of spherical shaped fullerene. The calculated effective parameters were then used in our 3D heat transfer model to introduce the thermal conductivity of paraffin structures.

Simulated maximum temperature rises for the battery pack for different charging/discharging C-rates are depicted in Figure 5. As the first finding, we could observe remarkable decline in temperature rises by the use of PCM materials for all applying C-rates. However, in all studied cases, fabrication of paraffin nanocomposites does not show huge effect on the temperature damping in comparison with pure paraffin. It is worthy to note that in the electrochemical simulations in this study, we considered stop conditions of $4.8 \mathrm{~V}$ and $3.0 \mathrm{~V}$ for charging and discharging cycles, respectively. This way, by increasing the C-rate, the simulations were stopped in much earlier times because of reaching the stopping criteria. So, the temperature rises decrease by increasing the $\mathrm{C}$-rate because the heat generation was achieved in a much shorter time. From the theoretical point of view, by increasing the battery current, the reaction and ohmic heats ((13) and (14)) increase by the power of two while reversible heats increase linearly. On the other hand, the simulation results in Figure 5 also show that during discharging process the temperature rises are approximately twice the ones during the charging process. This is due to the fact that during the charging process reversible heat generated in the positive $\mathrm{LiCoO}_{2}$ electrode is negative which results in the cooling of the battery. However, during the discharging process the reversible heating in the positive electrode is mainly positive leading to the heating of the batteries. It should be emphasized that based on the $\partial U / \partial T$ curves reported in [2], the reversible heating is mainly dominated by the positive $\mathrm{LiCoO}_{2}$ electrode compared with MCMB negative electrode.

Simulation results for the maximum temperature rises for the battery pack with the use of paraffin nanocomposites with different concentrations of nanofillers during a 1C current for a single charging (a) and discharging (b) cycle are illustrated in Figure 6. As expected, by increasing the nanofiller's concentration inside the paraffin, the maximum temperature rises in the battery decrease continuously. In the discharging cycles, the maximum temperature rise decreases by around $3 \mathrm{~K}$ through the addition of $5 \%$ volume ratio of CNT fillers inside the paraffin which is a remarkable enhancement. This temperature decrease might be crucial in applications in which the performance of the building blocks is sensitive to the temperature changes such as in nanoelectronics. Our modeling results suggest that fabrication of paraffin nanocomposites as a PCM for the heat management of batteries could be considered as a solution if the battery is supposed to work under fast and continuous discharging cycles. Nonetheless, our simulation results clearly confirm the importance of the use of paraffin based PCMs for 


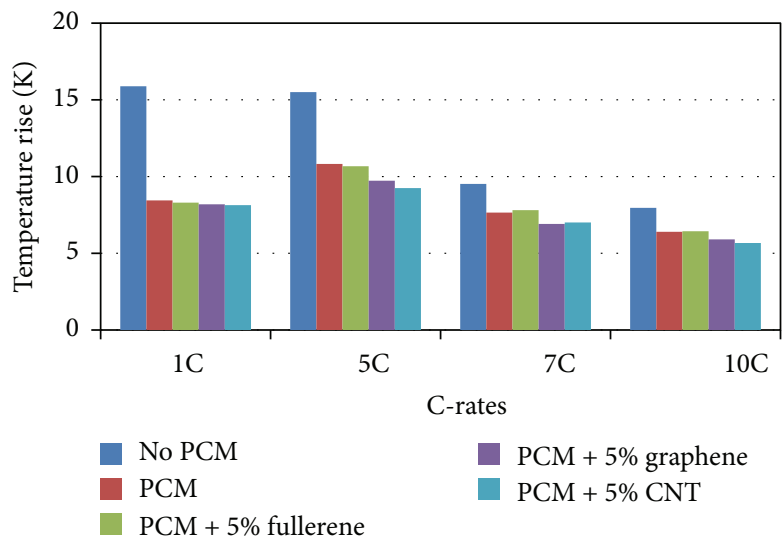

(a)

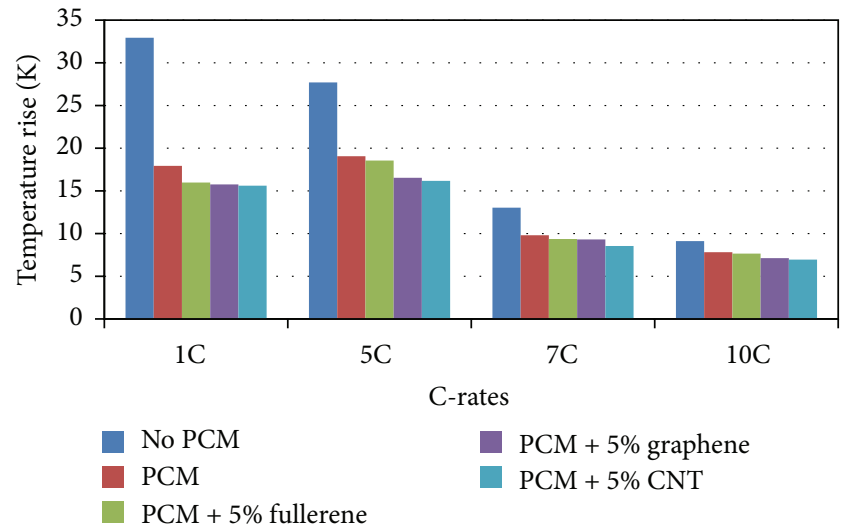

(b)

FIGURE 5: Simulated temperature rises for the battery pack for different charging (a) and discharging (b) C-rates.

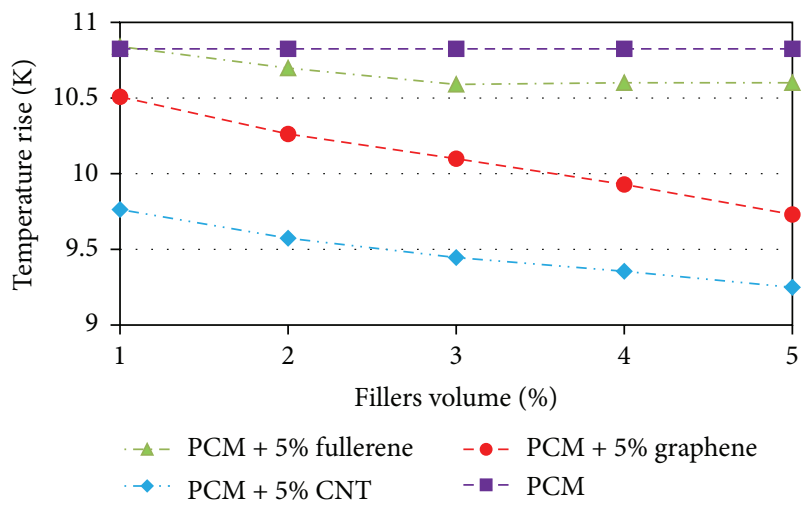

(a)

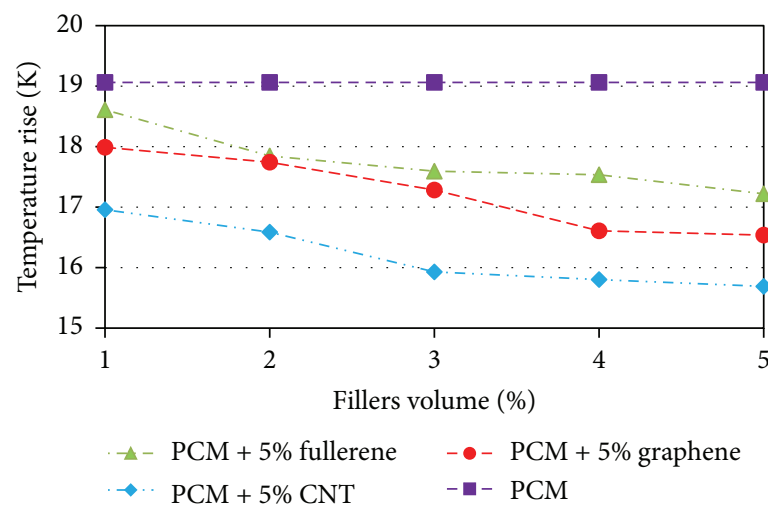

(b)

FIGURE 6: Modeling result for the maximum temperature rises for the battery pack with the use of paraffin nanocomposites with different concentrations of nanofillers during a $1 \mathrm{C}$ current for a single charging (a) and discharging (b) cycle.

the thermal management of $\mathrm{Li}$-ion batteries for both charging and discharging cycles.

In Figure 7, two samples of calculated voltage curves and simulated 3D temperature profiles of the battery pack surrounded by a paraffin matrix mixed with $5 \%$ carbon nanotubes at different times during a $1 \mathrm{C}$ charging and discharging cycles are plotted. Interestingly, for the charging cycle at the time of 10 seconds we noticed that the battery pack temperature is lower than that of ambient. This means that reversible cooling due to the entropy changes that occurred in the positive electrode was large enough to surpass ohmic and reaction heat generations. The simulated temperature profiles reveal that regardless of the initial times of charging cycle, for the rest of times, the maximum temperature rises take place inside the batteries somewhere close to the center of the battery pack. Therefore, one solution to improve the temperature management of the battery pack is to include higher concentration of the fillers within the paraffin materials in the sections that are closer to the center of the pack in order to enhance the heat conduction to the surroundings. Consequently, our modeling suggests that the heat management of a battery pack can be improved by an appropriate use of paraffin nanocomposites in the positions where the maximum temperature rises occur.

\section{Conclusion}

We developed an electrochemical model based on Newman's pseudo-2D model coupled with a 3D heat transfer model to investigate the heat management of a battery pack. The considered battery is made of $\mathrm{LiCoO}_{2}$ positive composite electrode and mesocarbon microbead negative electrode. Based on the electrochemical theory, the heat generation during charging/discharging cycles was calculated which was used in the $3 \mathrm{D}$ heat transfer model for the evaluation of temperature rises in a battery pack under various loading conditions. Our modelling results revealed remarkable decrease in temperature rises by embedding the batteries inside a paraffin wax. This effect was found to be more considerable for discharging cycles. Then, we studied the effects of fabrication of paraffin nanocomposites on temperature rises of a battery pack. We included different carbon based nanofillers such as graphene, carbon nanotubes, and fullerene for the improvement of thermal conduction of paraffin. The thermal conductivity 


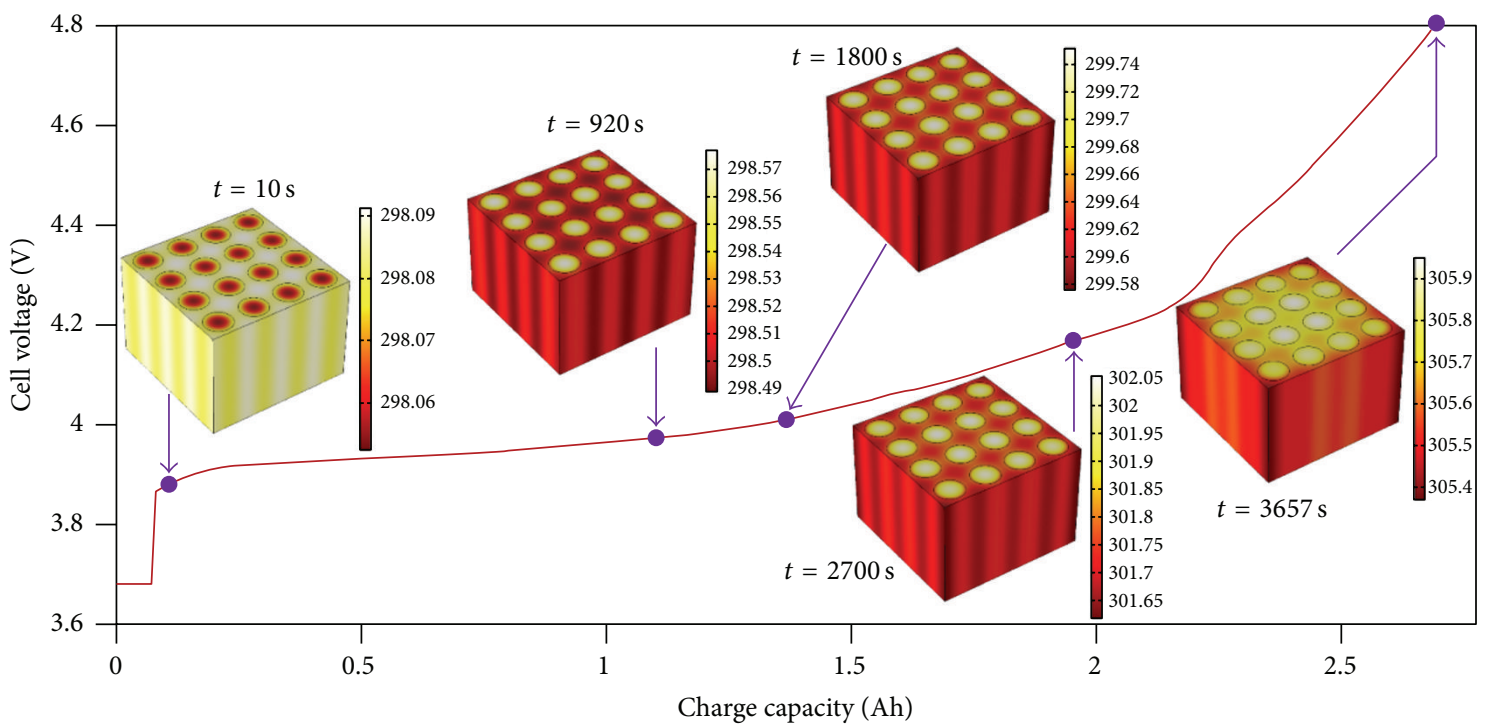

(a)

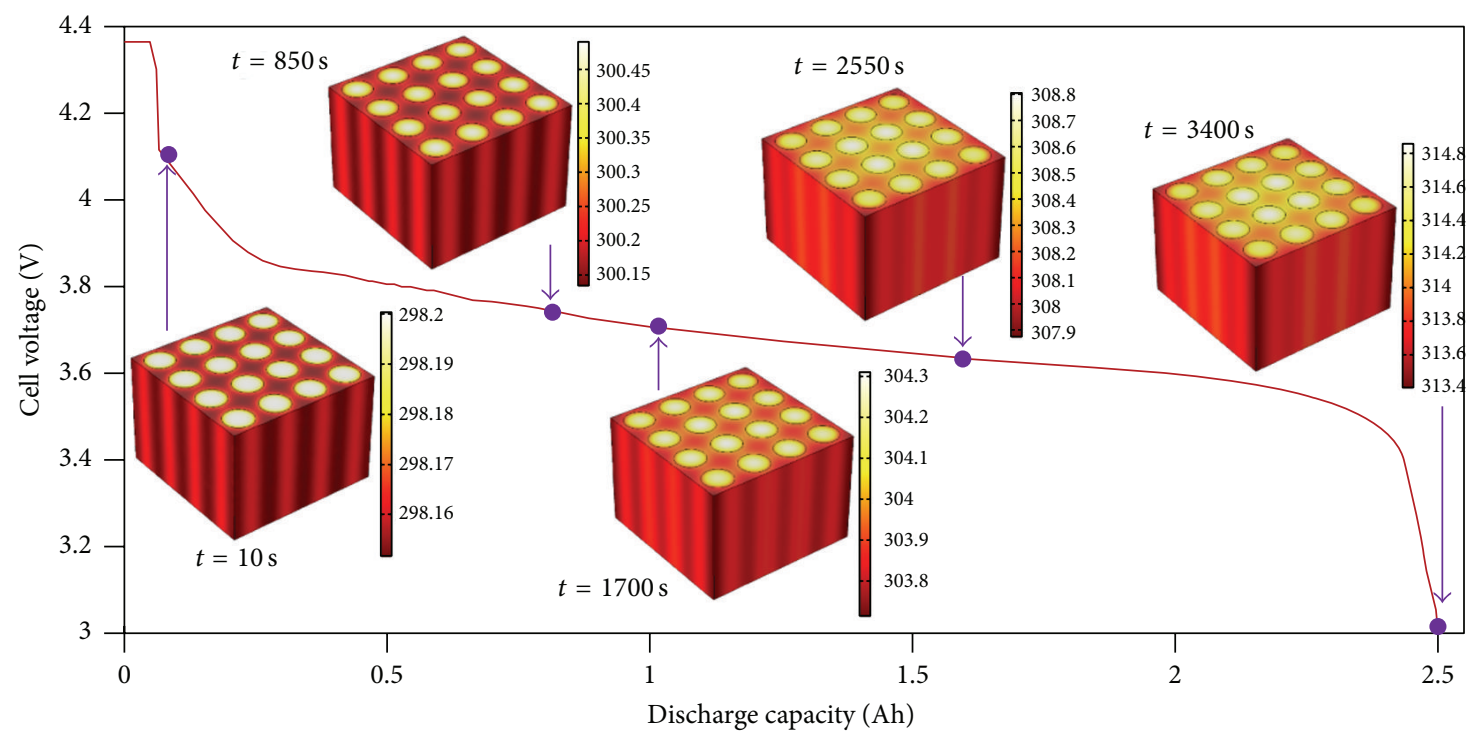

(b)

FIGURE 7: Calculated voltage curves and simulated 3D temperature profiles at various times during a 1C charging (a) and discharging (b) cycle. The ambient temperature was set to the room temperature (298.15 K).

for all fillershas been considered to be the same so that our emphasis in the present study is related to the effect of fillers geometry. The effective thermal conductivity of paraffin nanocomposites was evaluated using advanced 3D finite element models. It is shown that the fabrication of paraffin nanocomposites can increase the enhancement of the heat management of batteries. In this case, a favorable case was found to be the paraffin wax that was enhanced by the carbon nanotubes. We note that we did not include the effect of temperature on the thermal properties of paraffin structures; however, a separate investigation can be yet conducted in this direction. It was shown that during the charging process, the reversible heat generated in the positive $\mathrm{LiCoO}_{2}$ resulted in the cooling of the battery so that there is a remarkably lower temperature rises compared to discharging cycles. Our modeling results suggest that fabrication of paraffin nanocomposites for the heat management of batteries can be considered as a promising solution if the battery is to be work under fast and continuous discharging cycles. In summary, the proposed modeling methodology can be considered as an efficient method for the design of Li-ion batteries packs with enhanced heat management. In the future, we intend to extend our framework to a multiscale approach accounting also for the fine scale features [31-35] of the batteries which 
is of utmost importance for the design of new battery materials.

\section{Conflict of Interests}

The authors declare that there is no conflict of interests regarding the publication of this paper.

\section{Acknowledgment}

The authors greatly acknowledge the financial support by European Union through ERC grant for COMBAT project.

\section{References}

[1] P. Goli, S. Legedza, A. Dhar, R. Salgado, J. Renteria, and A. A. Balandin, "Graphene-enhanced hybrid phase change materials for thermal management of Li-ion batteries," Journal of Power Sources, vol. 248, pp. 37-43, 2014.

[2] K. Kumaresan, G. Sikha, and R. E. White, "Thermal model for a Li-ion cell," Journal of the Electrochemical Society, vol. 155, no. 2, pp. A164-A171, 2008.

[3] V. Goyal and A. A. Balandin, "Thermal properties of the hybrid graphene-metal nano-micro-composites: applications in thermal interface materials," Applied Physics Letters, vol. 100, Article ID 073113, 2012.

[4] M. D. Zolot, K. Kelly, M. Keyser, M. Mihalic, and A. Pesaran, "Thermal evaluation of the Honda insight battery pack," in Proceedings of the 36th Intersociety Energy Conversion Engineering Conference, Savannah, Ga, USA, June 2001.

[5] K. Yeow, H. Teng, M. Thelliez, and E. Tan, "3D thermal analysis of Li-ion battery cells with various geometries and cooling conditions using abaqus," in Proceedings of the SIMULIA Community Conference, Providence, RI, USA, May 2012.

[6] R. Benger, H. Wenzl, H.-P. Beck, M. Jiang, D. Ohms, and G. Schaedlich, "Electrochemical and thermal modeling of lithiumion cells for use in HEV or EV application," World Electric Vehicle Journal, vol. 3, no. 1, pp. 342-351, 2009.

[7] S. K. Mohammadian, Y. He, and Y. Zhang, "Internal cooling of a lithium-ion battery using electrolyte as coolant through microchannels embedded inside the electrodes," Journal of Power Sources, vol. 293, pp. 458-466, 2015.

[8] A. Sharma, V. V. Tyagi, C. R. Chen, and D. Buddhi, "Review on thermal energy storage with phase change materials and applications," Renewable and Sustainable Energy Reviews, vol. 13, no. 2, pp. 318-345, 2009.

[9] S. Ghosh, W. Bao, D. L. Nika et al., "Dimensional crossover of thermal transport in few-layer graphene," Nature Materials, vol. 9, no. 7, pp. 555-558, 2010.

[10] K. M. F. Shahil and A. A. Balandin, "Graphene-multilayer graphene nanocomposites as highly efficient thermal interface materials," Nano Letters, vol. 12, no. 2, pp. 861-867, 2012.

[11] B. Mortazavi, J. Bardon, and S. Ahzi, "Interphase effect on the elastic and thermal conductivity response of polymer nanocomposite materials: 3D finite element study," Computational Materials Science, vol. 69, pp. 100-106, 2013.

[12] N. Sheng, M. C. Boyce, D. M. Parks, G. C. Rutledge, J. I. Abes, and R. E. Cohen, "Multiscale micromechanical modeling of polymer/clay nanocomposites and the effective clay particle," Polymer, vol. 45, no. 2, pp. 487-506, 2004.
[13] Q. H. Zeng, A. B. Yu, and G. Q. Lu, "Multiscale modeling and simulation of polymer nanocomposites," Progress in Polymer Science, vol. 33, no. 2, pp. 191-269, 2008.

[14] D. Luo, W.-X. Wang, and Y. Takao, "Effects of the distribution and geometry of carbon nanotubes on the macroscopic stiffness and microscopic stresses of nanocomposites," Composites Science and Technology, vol. 67, no. 14, pp. 2947-2958, 2007.

[15] J. D. Renteria, D. L. Nika, and A. A. Balandin, "Graphene thermal properties: applications in thermal management and energy storage," Applied Sciences, vol. 4, no. 4, pp. 525-547, 2014.

[16] J. Renteria, S. Legedza, R. Salgado et al., "Magneticallyfunctionalized self-aligning graphene fillers for high-efficiency thermal management applications," Materials \& Design, vol. 88, pp. 214-221, 2015.

[17] J. D. Renteria, S. Ramirez, H. Malekpour et al., "Strongly anisotropic thermal conductivity of free-standing reduced graphene oxide films annealed at high temperature," Advanced Functional Materials, vol. 25, no. 29, pp. 4664-4672, 2015.

[18] A. A. Khatibi and B. Mortazavi, "A study on the nanoindentation behaviour of single crystal silicon using hybrid MD-FE method," Advanced Materials Research, vol. 32, pp. 259-262, 2008.

[19] B. Mortazavi, A. A. Khatibi, and C. Politis, "Molecular dynamics investigation of loading rate effects on mechanical-failure behaviour of FCC metals," Journal of Computational and Theoretical Nanoscience, vol. 6, no. 3, pp. 644-652, 2009.

[20] J. Newman and K. E. Thomas-Alyea, Electrochemical Systems, John Wiley \& Sons, Prentice-Hall, Eaglewood Cliffs, NJ, USA, 3rd edition, 2004.

[21] M. Guo and R. E. White, "A distributed thermal model for a Liion electrode plate pair," Journal of Power Sources, vol. 221, pp. 334-344, 2013.

[22] K. Smith and C.-Y. Wang, "Power and thermal characterization of a lithium-ion battery pack for hybrid-electric vehicles," Journal of Power Sources, vol. 160, no. 1, pp. 662-673, 2006.

[23] L. Cai and R. E. White, "Mathematical modeling of a lithium ion battery with thermal effects in COMSOL Inc. Multiphysics (MP) software," Journal of Power Sources, vol. 196, no. 14, pp. 5985-5989, 2011.

[24] B. Wu, V. Yufit, M. Marinescu, G. J. Offer, R. F. MartinezBotas, and N. P. Brandon, "Coupled thermal-electrochemical modelling of uneven heat generation in lithium-ion battery packs," Journal of Power Sources, vol. 243, pp. 544-554, 2013.

[25] M. Sievers, U. Sievers, and S. S. Mao, "Thermal modelling of new Li-ion cell design modifications," Forschung im Ingenieurwesen, vol. 74, no. 4, pp. 215-231, 2010.

[26] H. Maleki, S. Al Hallaj, J. R. Selman, R. B. Dinwiddie, and H. Wang, "Thermal properties of lithium-ion battery and components," Journal of the Electrochemical Society, vol. 146, no. 3, pp. 947-954, 1999.

[27] A. H. N. Shirazi, M. R. Azadi Kakavand, and T. Rabczuk, "Numerical study of composite electrode's particle size effect on the electrochemical and heat generation of a Li-ion battery," Journal of Nanotechnology in Engineering and Medicine, In press.

[28] B. Mortazavi, F. Hassouna, A. Laachachi et al., "Experimental and multiscale modeling of thermal conductivity and elastic properties of PLA/expanded graphite polymer nanocomposites," Thermochimica Acta, vol. 552, pp. 106-113, 2013.

[29] B. Mortazavi, O. Benzerara, H. Meyer, J. Bardon, and S. Ahzi, "Combined molecular dynamics-finite element multiscale modeling of thermal conduction in graphene epoxy nanocomposites," Carbon, vol. 60, pp. 356-365, 2013. 
[30] A. A. Balandin, "Thermal properties of graphene and nanostructured carbon materials," Nature Materials, vol. 10, no. 8, pp. 569-581, 2011.

[31] P. R. Budarapu, R. Gracie, S. P. A. Bordas, and T. Rabczuk, "An adaptive multiscale method for quasi-static crack growth," Computational Mechanics, vol. 53, no. 6, pp. 1129-1148, 2014.

[32] P. R. Budarapu, R. Gracie, S.-W. Yang, X. Zhuang, and T. Rabczuk, "Efficient coarse graining in multiscale modeling of fracture," Theoretical and Applied Fracture Mechanics, vol. 69, pp. 126-143, 2014.

[33] H. Talebi, M. Silani, S. P. A. Bordas, P. Kerfriden, and T. Rabczuk, "Molecular dynamics/xfem coupling by a threedimensional extended bridging domain with applications to dynamic brittle fracture," International Journal for Multiscale Computational Engineering, vol. 11, no. 6, pp. 527-541, 2013.

[34] H. Talebi, M. Silani, S. Bordas, P. Kerfriden, and T. Rabczuk, "A computational library for multiscale modeling of material failure," Computational Mechanics, vol. 53, no. 5, pp. 1047-1071, 2014.

[35] H. Talebi, M. Silani, and T. Rabczuk, "Concurrent multiscale modeling of three dimensional crack and dislocation propagation," Advances in Engineering Software, vol. 80, pp. 82-92, 2015. 

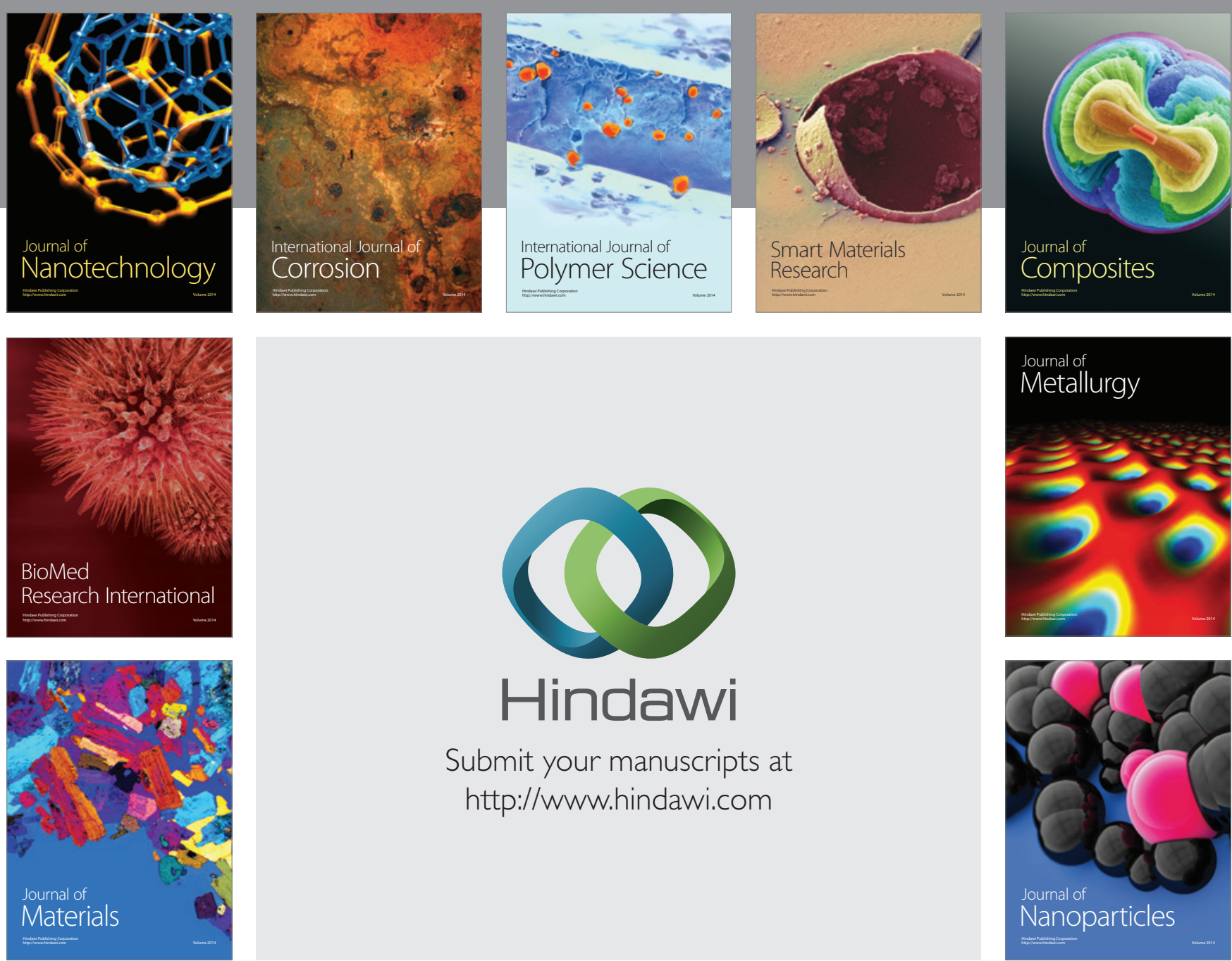

\section{Hindawi}

Submit your manuscripts at

http://www.hindawi.com

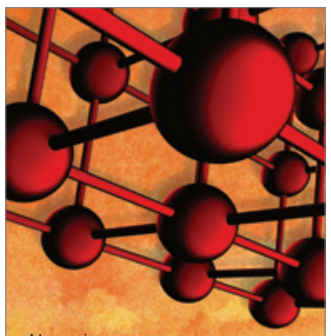

Materials Science and Engineering
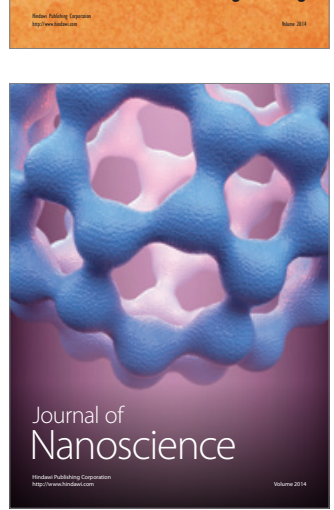
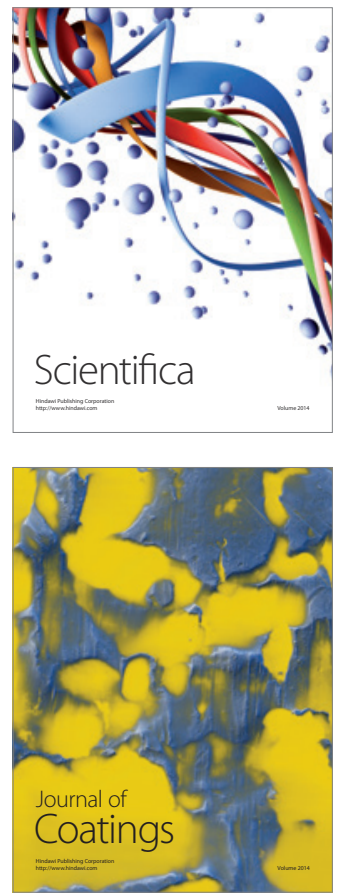
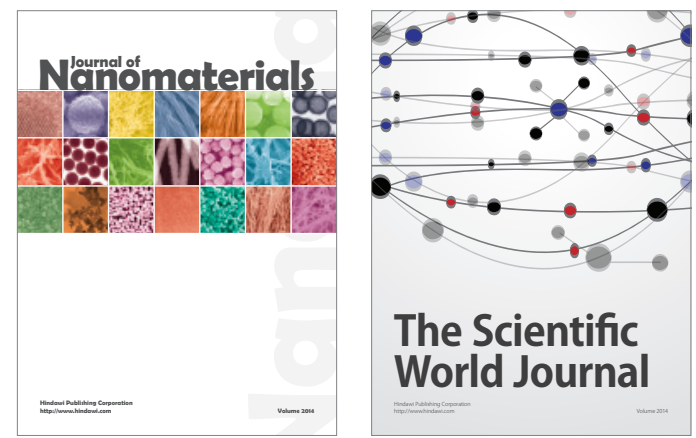

The Scientific World Journal
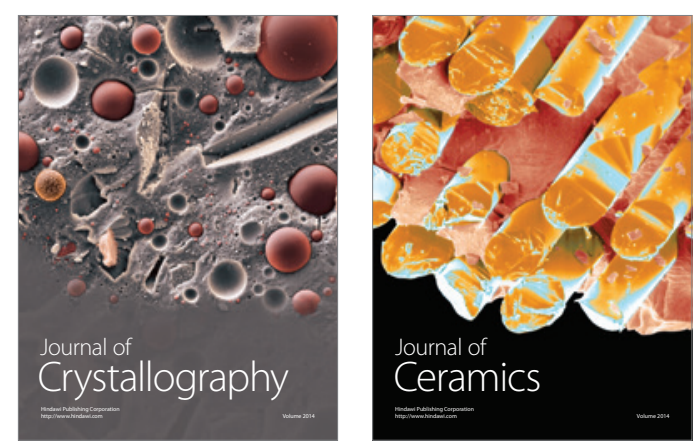
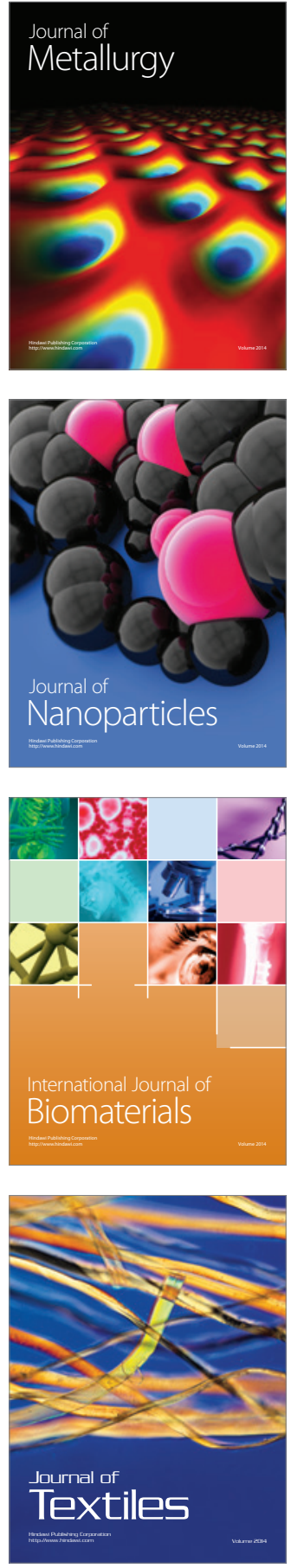